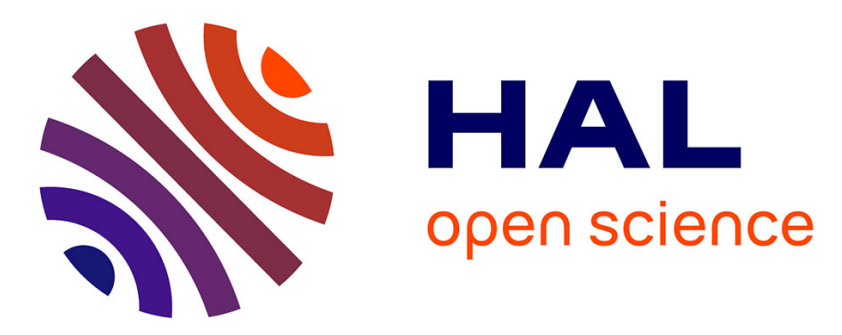

\title{
Weakly Calibrated Stereoscopic Visual Servoing for Laser Steering: Application to Phonomicrosurgery.
}

Brahim Tamadazte, Nicolas Andreff

\section{To cite this version:}

Brahim Tamadazte, Nicolas Andreff. Weakly Calibrated Stereoscopic Visual Servoing for Laser Steering: Application to Phonomicrosurgery.. IEEE/RSJ International COnference on Intelligent Robots and Systems, IROS 2014., Sep 2014, Chicago, IL, United States. pp.743-748, 10.1109/IROS.2014.6942641 . hal-01313483

\section{HAL Id: hal-01313483 \\ https://hal.science/hal-01313483}

Submitted on 10 May 2016

HAL is a multi-disciplinary open access archive for the deposit and dissemination of scientific research documents, whether they are published or not. The documents may come from teaching and research institutions in France or abroad, or from public or private research centers.
L'archive ouverte pluridisciplinaire HAL, est destinée au dépôt et à la diffusion de documents scientifiques de niveau recherche, publiés ou non, émanant des établissements d'enseignement et de recherche français ou étrangers, des laboratoires publics ou privés. 


\title{
Weakly Calibrated Stereoscopic Visual Servoing for Laser Steering: Application to Phonomicrosurgery
}

\author{
Brahim Tamadazte and Nicolas Andreff
}

\begin{abstract}
This paper deals with the study of a weakly calibrated multiview visual servoing control law for microrobotic laser phonomicrosurgery of the vocal folds. It consists of the development of an endoluminal surgery system for laser ablation and resection of cancerous tissues. More specifically, this paper focuses on the part concerning the control of the laser spot displacement during surgical interventions. To perform this, a visual control law based on trifocal geometry is designed using two cameras and a laser source (virtual camera). The method is validated on a realistic testbench and the straight point-to-point trajectories are demonstrated.
\end{abstract}

\section{INTRODUCTION}

The direct diagnosis of the larynx and the trachea are essential in the Otolaryngology field. This is possible using multiple existing endoscopic vision systems. The first endoscopic system for larynx inspection is probably the glottoscope developed by [1], in 1828 (Fig. 1(a)). Since then, these systems have largely evolved towards more efficient inspection and surgery intervention devices. The most successful protocol for surgery of vocal folds, widely used in hospitals is certainly the suspension laryngoscopic technique which consists of a straight-rigid laryngoscope, a stereomicroscope, a set of specific instruments, a laser source, and a foot-pedal controlled device to activate the laser [2]. These systems allow performing more precise diagnostics and microsurgeries despite many drawbacks for patients and surgeons. For instance, the AcuBlade ${ }^{\mathrm{TM}}$ system which claims to be a robotic system uses a stereomicroscope and a laser source placed outside, at $400 \mathrm{~mm}$ from the vocal cords (Fig. 1(b)). This disposition involves the projection, in straight line, of the laser beam on the soft tissue through the patient's mouth and the laryngoscope tunnel. This causes a very uncomfortable position for the patient with an extreme extension of the neck, that makes it painful several days after the operation [3]. Apart from this, the requirement of dexterity and considerable expertise of the surgeon to perform an intervention of the vocal cords are highly required. This is true, among other, because the laser displacements are performed in open-loop control.

In our work, we propose a new concept of a more intuitive adjustable laryngoscope for microrobotic laser phonosurgery of the vocal cords. This consists of an endoscope which is

This work was supported by $\mu$ RALP, the EC FP7 ICT Collaborative Project no. 288663 (http://www.microralp.eu), and by ACTION, the French ANR Labex no. ANR-11-LABX-01-01 (http://www. labex-action.fr).

The authors are with FEMTO-ST Institute, AS2M department, Université de Franche-Comté/CNRS/ENSMM/UTBM, 24 rue Savary, 25000 Besanọn, France. name. namedfemto-st. fr equipped with a two degrees-of-freedom (dof) micromirror, a fixed mirror, two image bundles, a lighting source, and a laser source (Fig. 1(c)). This paper focuses specifically on the design of a closed-loop control strategy to achieve automatic laser partial ablation or resection of local cancerous tissues. Using a closed-loop visual feedback control law, commonly called visual servoing, is justified by its robustness to calibration errors (camera and robot) and to changes in the robot's environment [4]. The proposed visual servoing control law is based on the use of the multiview geometrical constraints. Indeed, with two real cameras and one virtual camera (i.e., if we consider the laser beam as a virtual camera), it is possible to build a trifocal geometry system. Thereby, using the trifocal constraint, it is possible to design an original visual servoing control law.

Visual servoing for visible laser control has been widely studied in the literature for many years. This covers several applications in industrial [5], [6], mobile [7], [8] or surgical robotics [9]. The laser scanner or pointer is generally used in two configurations: eye-to-hand [10] or eye-in-hand [5] according to the position of the laser with respect to the camera(s). An exception is [13] where a laser scan was rigidly attached to a camera. Thus, the authors considered the camera+laser system as degenerate stereoscopic system to control the depth directly by servoing the laser spot position on its epipolar line.

In all cases, the laser is considered as an additional sensor for the estimation of scene depth. Extending this idea, we propose to consider the moving laser as a virtual camera grabbing one pixel at a time. This allowed us to take into account the epipolar geometry constraint generated by the stereoscopic pair camera/laser in the design of more efficient visual servoing control laws [11]. Epipolar geometry-based visual servoing was efficiently investigated in the past [12], [14]-[16]. This type of control has also been successfully used for surgical applications [17], [18]. However, the more interesting idea for visual servoing applications is to use three views instead of two views. This is commonly known under the term trifocal system [19]. Some authors have addressed this problem in control approaches by coupling multiview geometry and visual servoing, for example using the trifocal constraint between two successive stereoscopic pairs [20] or between the initial, current and desired monocular views [21].

In this paper, the two-views and one laser system is considered as an analogous system to a degenerate case of trifocal geometry (Fig. 2). Thereby, it is possible to design original trifocal-based visual servoing approach simply using 


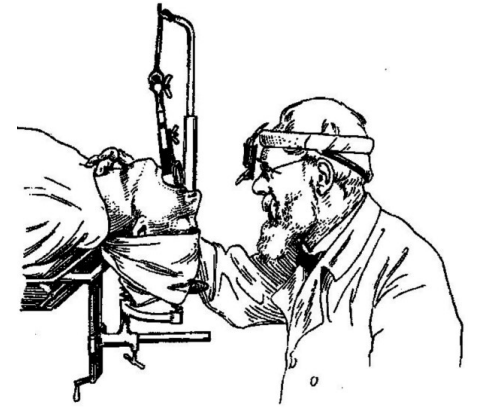

(a) Old system.

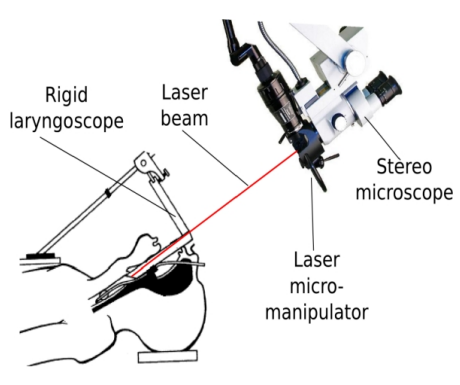

(b) Current system.

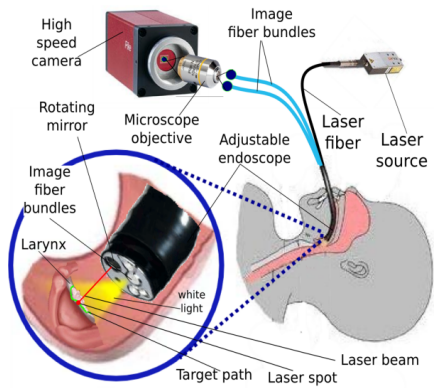

(c) Targeted system.

Fig. 1. Glottoscope in the past, today, and in the future.

the geometric properties of the three-view system. It will be shown that this approach has several advantages in terms of robustness, behavior, convergence or numerical complexity over the traditional approaches.

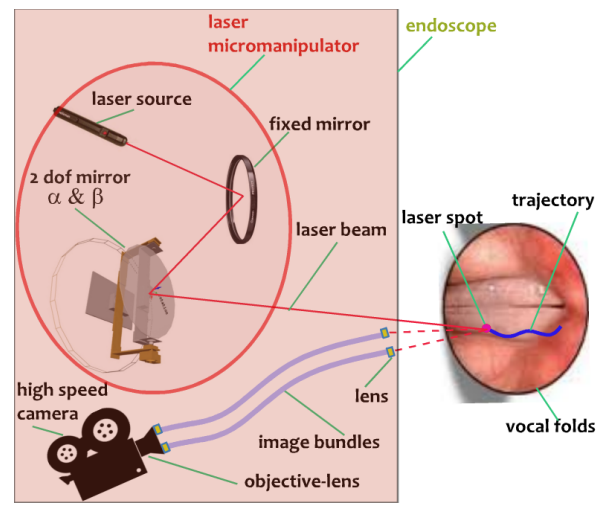

Fig. 2. Schematic view of the laser steering system with two cameras.

This paper is structured as follows: in Section II, trifocal geometry principles are provided and Section III deals with the proposed analogy of our system with the traditional trifocal system. Section IV shows design of the trifocal geometry based control law. Section V presents the experimental set-up and the validation results.

\section{TRIFOCAL GEOMETRY}

\section{A. Notations}

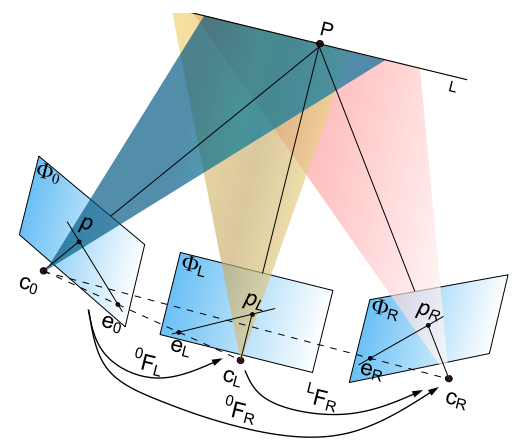

Fig. 3. Trifocal geometry principle.
For a better understanding of different notations introduced in this paper, the reader may refer to the following Table 1.

\begin{tabular}{c|l}
\hline Notations & Description \\
\hline $\mathbf{I}_{L}$ and $\mathbf{I}_{R}$ & left image and right image, respectively \\
$\tilde{p}_{L}$ and $\tilde{p}_{R}$ & 2D homogeneous points projected in $\mathbf{I}_{L}$ and $\mathbf{I}_{R}$ \\
${ }^{0} \tilde{\mathbf{P}}$ & 3D homogeneous point in the world frame ${ }^{0} R$ \\
$\tilde{p}$ & projection of ${ }^{0} \mathbf{P}$ in the image $\mathbf{I}$ \\
$\tilde{p}^{*}$ and $\dot{\tilde{p}}^{*}$ & desired image position and feed-forward term \\
${ }^{i} \mathbf{F}_{j}$ & fundamental matrix between the cameras $i$ and $j$ \\
$\mathcal{T}$ & a $3 \times 3 \times 3$ trifocal tensor \\
$\mathbf{T}_{i}$ & one of the three $3 \times 3$ matrix of $\mathcal{T}$ \\
${ }^{0} \underline{z}$ & $z$ direction of the laser beam from the \\
${ }^{0} z^{*}$ & mobile mirror towards the vocal folds \\
${ }^{0}$ & desired direction of the laser beam \\
$\underline{d}$ & time-derivation of ${ }^{0} z$ \\
$Z$ & distance between the mobile mirror and the vocal folds \\
$h_{R}$ & depth information of the scene \\
$h_{R} h_{L}$ & normal vector to the epipolar plans \\
$\mathbf{D}$ & differential kinematic matrix of the mirror \\
$\boldsymbol{\omega}$ & rotation velocity of the mirror \\
\hline
\end{tabular}

TABLE I

NOTATIONS.

Let three cameras with optical centers $c_{0}, c_{L}$ and $c_{R}$ observe a 3D point ${ }^{0} \mathbf{P}=(X, Y, Z)^{\top}$ which is projected in 2D points $p=(x, y)^{\top}, p_{L}=\left(x_{L}, y_{L}\right)^{\top}$, and $p_{R}=\left(x_{R}, y_{R}\right)^{\top}$ in the images planes $\Phi_{0}, \Phi_{L}$, and $\Phi_{R}$, respectively. The lines $\left(c_{0} c_{L}\right)$, and $\left(c_{0} c_{R}\right)$ define the baselines between each pair (camera, laser). The intersections of these baselines with the image planes give the epipole points $e_{0}, e_{L}$, and $e_{R}$. Also, $\left(e_{0} p\right),\left(e_{L} p_{L}\right)$, and $\left(e_{R} p_{R}\right)$ are called epipolar lines (Fig. 3). There are mathematical relations between the epipolar lines $\left(e_{0} p\right),\left(e_{L} p_{L}\right)$, and $\left(e_{R} p_{R}\right)$ and the 2D point $p$, commonly called epipolar constraint which are given by:

$$
\begin{aligned}
\tilde{p}^{\top 0} \mathbf{F}_{L} \tilde{p}_{L} & =0 \\
\tilde{p}_{L}^{\top L} \mathbf{F}_{R} \tilde{p}_{R} & =0 \\
\tilde{p}^{0} \mathbf{F}_{R} \tilde{p}_{R}^{\top} & =0
\end{aligned}
$$

where ${ }^{i} \mathbf{F}_{j}$ is the fundamental matrix between camera $i$ and camera $j$ and $\tilde{p}_{i}$ the homogenous representation of $p_{i}\left(\tilde{p}_{i}=\right.$ $\left.\left(p_{i}, 1\right)^{\top}\right)$.

It is also possible to represent these constraints by the trifocal tensor of a point-point-point transformation de- 
fined [19] by:

$$
\left[\tilde{p}_{L}\right]_{\times}\left(\sum_{i=1}^{3} \tilde{p}_{i} \mathbf{T}_{i}\right)\left[\tilde{p}_{R}\right]_{\times}=0_{3 \times 3}
$$

where $\times$ is the cross-product and the set of $3 \times 3$ matrix $\mathbf{T}_{1}$, $\mathbf{T}_{2}$, and $\mathbf{T}_{3}$ represent the trifocal tensor $\mathcal{T}_{3 \times 3 \times 3}$. Therefore, it is possible to write the point-point-point transformation as:

$$
\left[\tilde{p}_{R}\right]_{\times}\left(\tilde{p}_{i} \mathcal{T}_{i}^{j k}\right)\left[\tilde{p}_{R}\right]_{\times}=0_{3 \times 3}
$$

with $i, j, k \in[1 ; 3]$.

In the following section, by isolating the specific role of the virtual image, we introduce another way of writing these constraints, more appropriate for designing the trifocal geometry-based visual servoing.

\section{VIRTUAL TRIFOCAL GEOMETRY}

As shown in Fig. 4, it is possible to define an analogy between our system and the conventional three-view system in Fig. 3. The approach shown here is based on a vectorial writing of the visual servoing problem using the trifocal constraint.

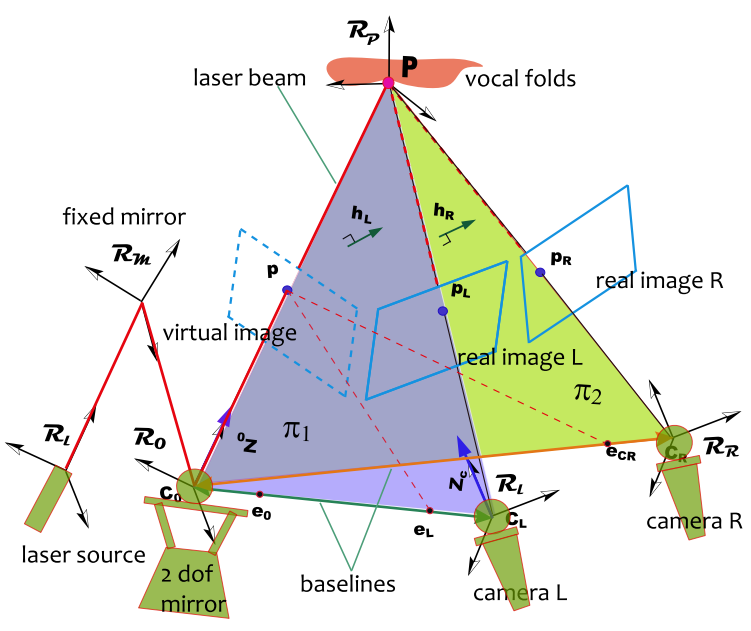

Fig. 4. Analogy with trifocal geometry.

Let us note ${ }^{0} \underline{z}$ the direction (unit vector) of the laser beam/line from the actuated mirror towards the target (tissue), ${ }^{0} \mathbf{P}$ the 3D position of the laser spot on the tissue, and $\tilde{p}$ its projection in the image. They are related by:

$$
{ }^{0} \mathbf{P}=d^{\mathbf{0}} \underline{z}=Z \tilde{p}
$$

with $d$ the distance between the mobile mirror and the surface, $\tilde{p}$ is the projection of ${ }^{0} \mathbf{P}$ on the virtual image and $Z$ the depth (i.e., the distance parallel to the virtual optical axis). It has to be noticed that neither the distance $d$ nor the depth $Z$ are easy to determine unlike the vector ${ }^{0} z$ that can obtained from the stereoscopic system, as shown later. We will not need $d$ and $Z$ any more in this paper.

From (6), it is easy to see that ${ }^{0} \underline{z}$ and $\tilde{p}$ are projectively the same. Therefore, it is possible to write:

$$
{ }^{0} \underline{z} \equiv \tilde{p}
$$

Actually, $\tilde{p}$ is the perspective projection on a plane, while ${ }^{0} \underline{z}$ is the perspective projection on a sphere.

\section{A. Trifocal Approach}

An original visual servoing control law can be derived using the trifocal constraint offered by our multiview vision system. Similar to the epipolar constraint, the three-view geometry can also be used to exploit the geometrical aspects in designing the control law. Therefore, from the layout shown in Fig. 4, it is possible to rewrite the trifocal constraint (1), (2), (3) and (7) in the following form:

$$
\begin{aligned}
\tilde{p}_{L}^{\top L} \mathbf{F}_{R} \tilde{p}_{R} & =0 \\
{ }^{0} z^{\top 0} \mathbf{F}_{R} \tilde{p}_{R} & =0 \\
{ }^{0} \underline{z}^{\top 0} \mathbf{F}_{L} \tilde{p}_{L} & =0
\end{aligned}
$$

Let us have aside (8) (except for improving the tracking of $\tilde{p}_{L}$ and $\tilde{p}_{R}$ ) and introduce $h_{R}$ and $h_{L}$ as:

$$
\begin{aligned}
h_{R} & ={ }^{0} \mathbf{F}_{R} \tilde{p}_{R} \\
h_{L} & ={ }^{0} \mathbf{F}_{L} \tilde{p}_{L}
\end{aligned}
$$

Consequently, from (9) and (10), it is trivial to see that $h_{R} \times h_{L}$ is parallel to ${ }^{0} \underline{z}$ (i.e. ${ }^{0} \underline{z} \equiv h_{R} \times h_{L}$ ). Using the "cross-product writing", it is allowed to write:

$$
{ }^{0} \underline{z} \times\left(h_{R} \times h_{L}\right)=0
$$

Note that this represents the trifocal constraint expressed in unbalanced manner, since ${ }^{0} \underline{z}$ is preferred.

\section{VISUAL SERVOING BASED ON VIRTUAL TRIFOCAL GEOMETRY}

\section{A. Kinematic Transformations}

The time derivative of (13) is:

$$
\begin{aligned}
& { }^{0} \underline{\dot{z}} \times\left(h_{R} \times h_{L}\right)+{ }^{0} \underline{z} \times\left(\left({ }^{0} \mathbf{F}_{R} \dot{\tilde{p}}_{R}\right) \times h_{L}\right) \\
& +{ }^{0} \underline{z} \times\left(h_{R} \times\left({ }^{0} \mathbf{F}_{L} \dot{\tilde{p}}_{L}\right)\right)=0
\end{aligned}
$$

which can be reorganized as:

$$
\begin{aligned}
& { }^{0} \underline{\dot{z}} \times\left(h_{R} \times h_{L}\right)= \\
& { }^{0} \underline{z} \times\left(h_{L} \times\left(\mathbf{F}_{R} \dot{\tilde{p}}_{R}\right)-h_{R} \times\left({ }^{0} \mathbf{F}_{L} \dot{\tilde{p}}_{L}\right)\right)
\end{aligned}
$$

From the trifocal constraint (13), we also get trivially:

$$
{ }^{0} \underline{z}=\frac{h_{R} \times h_{L}}{\left\|h_{R} \times h_{L}\right\|}
$$

because ${ }^{0} z$ is an unit vector. Hence, we have established a relationship between the velocities of the laser spot in each real image $\left(\dot{\tilde{p}}_{L}\right.$ and $\left.\dot{\tilde{p}}_{R}\right)$ and the laser beam change of orientation ${ }^{0} \underline{\dot{z}}$.

$$
\begin{aligned}
& { }^{0} \underline{\dot{z}} \times{ }^{0} \underline{z}= \\
& \frac{{ }^{0} \underline{z}}{\left\|h_{R} \times h_{L}\right\|} \times\left(h_{L} \times\left({ }^{0} \mathbf{F}_{R} \dot{\tilde{p}}_{R}\right)-h_{R} \times\left({ }^{0} \mathbf{F}_{L} \dot{\tilde{p}}_{L}\right)\right)
\end{aligned}
$$




\section{B. Proposed Control}

Now, it is necessary to link the laser beam velocities ${ }^{0} \underline{\dot{z}}$ to those of laser spot denoted $\boldsymbol{\omega}$ as follows:

$$
{ }^{0} \dot{z}=2 \omega \times{ }^{0} \underline{z}
$$

where, the factor 2 comes from the reflection law in optics in the mirror.

The analytic solution to (18) is:

$$
\boldsymbol{\omega}={ }^{0} \underline{z} \times{ }^{0} \underline{\dot{z}}+k^{0} \underline{z}, \quad k \in \mathfrak{R}
$$

However, a rotation of the laser beam around its axis does not have any use, and so, $k$ is chosen to be 0 .

Therefore, by identifying (17) and (19), we can easily deduce the expression of the new control law:

$$
\boldsymbol{\omega}=\frac{1}{2} \frac{{ }^{0} \underline{z}}{\left\|h_{R} \times h_{L}\right\|} \times\left(h_{L} \times\left(\mathbf{F}_{R} \dot{\tilde{p}}_{R}\right)-h_{R} \times\left({ }^{0} \mathbf{F}_{L} \dot{\tilde{p}}_{L}\right)\right)
$$

For an exponential decay of the cost-function, we introduce a first-order behavior of the error, in both images, between the current position $\tilde{p}$ and the desired position $\tilde{p}^{*}$ of the laser spot:

$$
\dot{\tilde{p}}_{i}=-\lambda\left(\tilde{p}_{i}-\tilde{p}_{i}^{*}\right)-\dot{\tilde{p}}_{i}^{*}, \quad i \in\{L, R\}
$$

where $\lambda$ is a positive gain and $\dot{\tilde{p}}_{i}^{*}$ is the feed-forward term in case of trajectory tracking which is directly given by the surgeon through a smart human-machine interface [22].

Replacing (21) in (20) yields:

$$
\begin{aligned}
\boldsymbol{\omega}= & -\lambda \kappa^{0} \underline{z} \times\left(h_{L} \times\left({ }^{0} \mathbf{F}_{R}\left(\tilde{p}_{R}-\tilde{p}_{R}^{*}\right)\right)-h_{R} \times\left({ }^{0} \mathbf{F}_{L}\left(\tilde{p}_{L}-\tilde{p}_{R}^{*}\right)\right)\right) \\
& -\kappa \times\left(h_{L} \times{ }^{0} \mathbf{F}_{R} \dot{\tilde{p}}_{R}^{*}-h_{R} \times{ }^{0} \mathbf{F}_{L} \dot{\tilde{p}}_{L}^{*}\right)
\end{aligned}
$$

where $\kappa=\frac{1}{\left\|h_{R} \times h_{L}\right\|}$.

Finally, $\boldsymbol{\omega}$ is converted to joint velocities $\dot{q}$ through the inverse differential kinematic matrix $\mathbf{D}^{-1}$ of the mirror mechanism as $\boldsymbol{\omega}=\mathbf{D}^{-1} \dot{q}$.

\section{Analysis}

It can be observed that the design of this new controller requires only vector calculus. This involves using the multiview geometry specificities in another innovative and trivial manner. As shown by the expression of the new visual servoing control law (22), it does not involve any of the following: i) matrix inversion; ii) prior knowledge of the scene structure; iii) camera nor Euclidean hand-eye calibration; iv) explicit 3D reconstruction nor estimation of the scene; v) proprioceptive sensing to know the mirror configuration. All the data is directly obtained from the images. The only parameters required are the fundamental matrices ${ }^{0} \mathbf{F}_{R}$ and ${ }^{0} \mathbf{F}_{L}$. In the following section, it will be shown that using a coarse estimation of these matrices will be sufficient and allows for a more than satisfactory behavior (robustness, convergence, precision, etc.) of the proposed control law.

\section{EXPERIMENTAL RESULTS}

\section{A. Experimental Set-up}

The proposed approach was tested on an experimental setup (Fig. 5) which consists of two CCD cameras characterized with a frame rate of $25 \mathrm{fps}$ and a resolution of $480 \times 640$ pixels for each, a laser source, a fixed mirror, an actuated mirror (S-334) from Physical Instruments Inc. The latter contains two single axis units $(\alpha$ and $\beta$ ) working in series with one common pivot point characterized by a bandwidth of $200 \mathrm{~Hz}$, a resolution of $0.2 \mu \mathrm{rad}$ and a motion range of $\pm 1.5^{\circ}$.

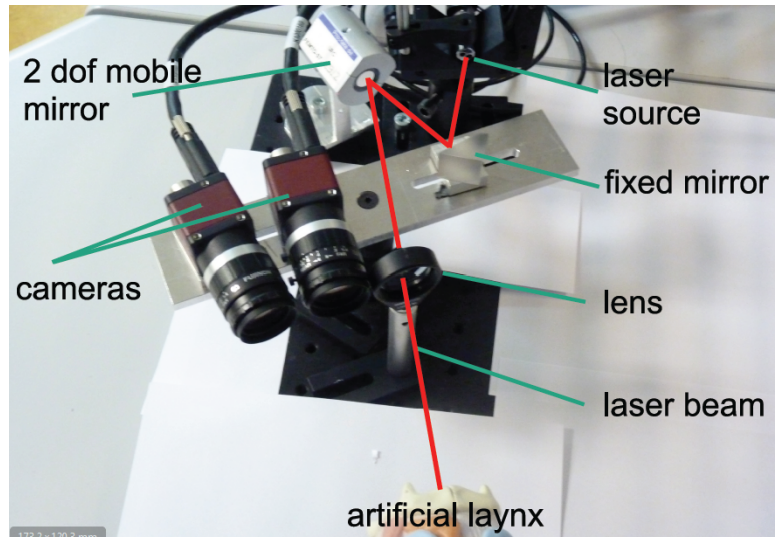

Fig. 5. Photography of the experimental set-up.

The current operational mode of the experimental set-up is as follows: the operator defines the desired position $\tilde{p}_{L}^{*}$ (using a mouse click) in $\mathbf{I}_{L}$, and using the fundamental matrix ${ }^{L} \mathbf{F}_{R}$ between the left camera and the right camera, it is possible to find the corresponding desired position $\tilde{p}_{R}^{*}$ in $\mathbf{I}_{R}$. Then, the time-varying positions $\tilde{p}_{L}$ and $\tilde{p}_{R}$ of the laser spot in both images are tracked using ViSP [23].

\section{B. Coarse Calibration}

It is important to note again that the results described in this section are obtained without any camera nor eye-to-hand calibration (i.e., left camera/mirror and right camera/mirror poses). The fundamental matrices ${ }^{0} \mathbf{F}_{L}$ (between the laser beam frame $R_{0}$ linked to the reflexion point in the mobile mirror and the left camera frame $R_{L}$ ), and ${ }^{0} \mathbf{F}_{R}$ (between $R_{0}$ and the right camera frame $R_{R}$ ) are approximated (estimated at hand) by a translation vector ${ }^{0} \mathbf{t}_{L, R}=(1,-0.1,38)^{\top} \mathrm{mm}$ and a rotation matrix ${ }^{0} \mathbf{R}_{L, R}=\mathbf{0}_{3 \times 3}$ and $\mathbf{D}$ identified in the image as $\mathbf{D}=\left[\begin{array}{cc}-10 & 0 \\ 0 & 10\end{array}\right]$.

\section{Results}

Not being able yet to test our developments directly on true vocal cords due to the lack of a technical solution for fiber imaging, we have imagined two scenarios. First, the surgical site consists of a planar scene on which the laser spot is projected. Second, the 2D scene is replaced by a 3D shape phantom. 

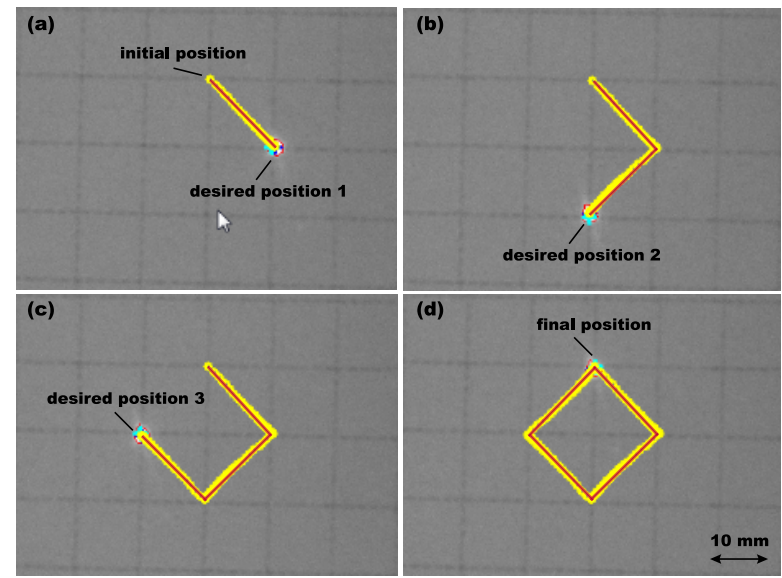

LEFT CAMERA

Fig. 6. Some images captured by the left camera which illustrate the displacement of the laser spot during the trifocal visual control. Superimposed lines are the performed trajectories and the ideal one.
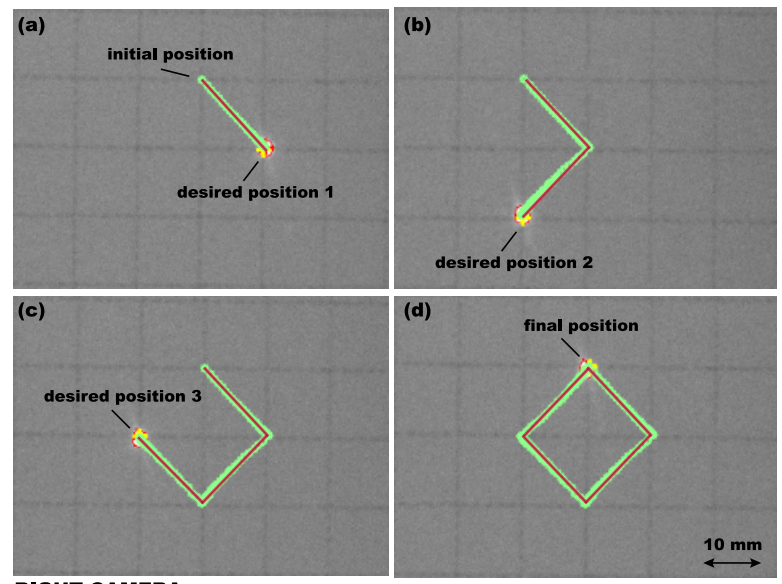

RIGHT CAMERA

Fig. 7. The same task shown in Fig. 6 viewed by the right camera

Fig. 6 and 7 show an image sequence captured, respectively, by the left camera and right camera during an automatic control of the laser spot displacements in a planar scene. Namely, Fig. 6(a) and Fig. 7(a) illustrate, simultaneously in $\mathbf{I}_{L}$ and $\mathbf{I}_{R}$, the achievement of the first subtask i.e., the steering of laser between the initial position $\tilde{p}_{L}$ and the first desired position $\tilde{p}_{L 1}^{*}$ in $\mathbf{I}_{L}$ (respectively, $\tilde{p}_{R}$ and $\tilde{p}_{R 1}^{*}$ in $\left.\mathbf{I}_{R}\right)$. When, the laser reaches the desired position, the user defines by a mouse-click the next desired position which the laser must attain. Then, Fig. 6(b) to (d) (respectively, Fig. 7(b) to (d)) represent the others subtasks performed by the controller. From the obtained results, it is clear that the laser spot reaches the desired position accurately. From this example, it can be noticed that the laser spot trajectory in both images is a straight line for a point-to-point displacement without any path planning phase. This means that our control law takes the shortest path both in images and space. This is the best achievable performance without having any rough estimation of the different calibration matrices.

It can be also noticed that the image errors in $\mathbf{I}_{L}$ follow an almost perfectly decoupled exponential decay to reach the desired position as shown in Fig. 8(a). It is the same in $\mathbf{I}_{R}$. The controller presents the same behavior concerning the velocities in $\mathbf{I}_{L}$ for $\alpha$ and $\beta$ (Fig. 8).
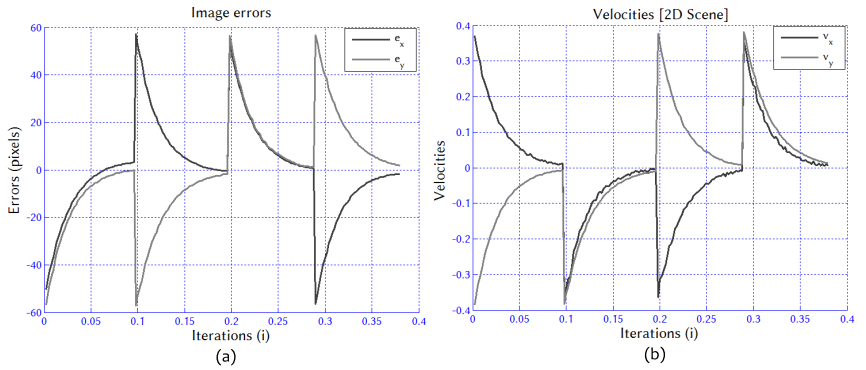

Fig. 8. (a) Image errors evolution $v s$. iterations $i$ and (b) velocities evolution $v s$. iterations $i\left[\mathbf{I}_{L}\right]$.

The laser steering is also validated in a scenario closer to the final application in order to study the performance of the controller in such conditions of use. To do this, we have manufactured a 3D shape surface (using a 3D printer) with hills of $5 \mathrm{~mm}$ of height. However, the validation task remains the same as in the $2 \mathrm{D}$ surface. Obviously, the idea is to observe the behavior of the laser spot displacements in this $3 \mathrm{D}$ object under the same conditions as in the first scenario i.e., the same set-up configuration and the coarse calibration parameters.
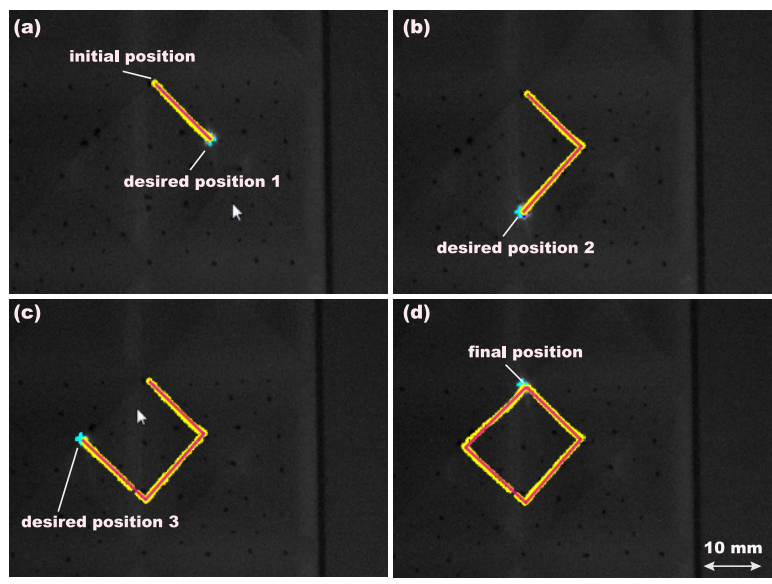

Fig. 9. Image sequence captured by the left camera during the laser steering on a 3D shape phantom. Superimposed lines are the performed trajectories and the point-to-point straight line.

The results of this second scenario are shown in Fig. 9 and 10 which represent some images captured by the left and the right cameras, respectively. They show the same task performed on a 3D surface. It can be seen that the laser spot point-to-point trajectories are very close to a straightline. This is performed without any prior knowledge nor 3D estimation (e.g., 3D reconstruction or depth computation) on the used 3D surface.

As in the case of 2D scene experiments, we can also notice the fact that the controller presents near perfect exponential convergence and decoupling behaviors. This is observed 

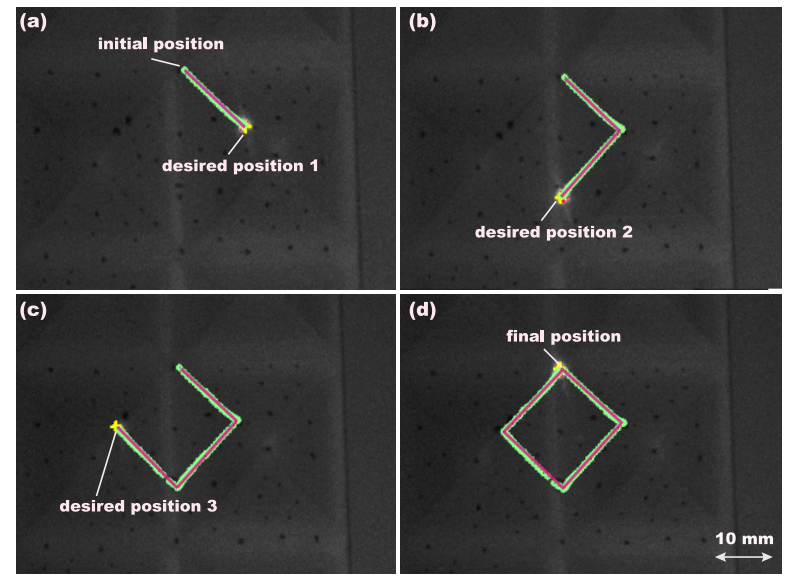

RIGHT CAMERA

Fig. 10. The same task shown in Fig. 9 viewed by the right camera.

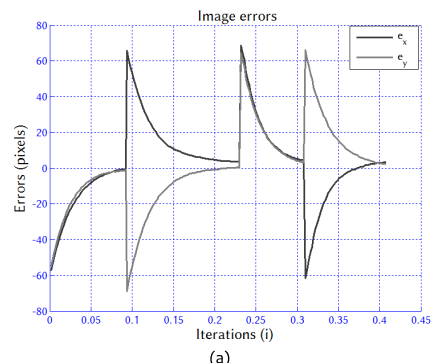

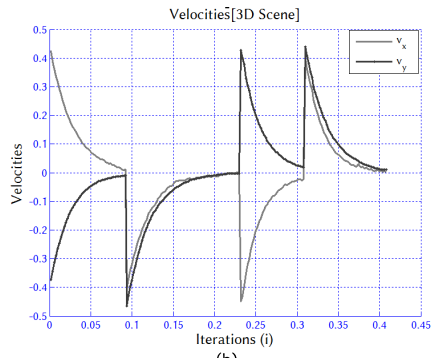

Fig. 11. (a) Image errors evolution vs. iterations $i$ and (b) velocities evolution vs. iterations $i\left[\mathbf{I}_{L}\right]$.

both image errors decay (Fig. 11(a)) and velocity evolutions (Fig. 11(b)).

\section{CONCLUSION AND FUTURE WORK}

In this paper, it was shown again that using multiview geometry simplifies the eye-to-hand visual control law. This is ultimately true in the case of laser steering applications. Laser surgery (e.g., laser phonomicrosurgery) often present this scenario, where a camera observes the laser spot displacements (scanning) on a 3D surface. Thereby, using the three-view constraint of our vision system, it was possible to design an optimal visual servoing control law without any calibration procedure (camera and eye-to-hand calibrations), nor explicit knowledge or reconstruction of the 3D scene. The proposed controller was tested in an experimental set-up to assess its relevance in terms of behavior and robustness. The obtained results have demonstrated that the proposed control law was fully decoupled (both image trajectories were straight) with an exponential decay of the image errors and also robust to calibration errors. Moreover, the proposed control law presents some other major advantages: i) no matrix inversion is required; ii) no proprioceptive sensor is needed on the micromirror; iii) no need of any prior knowledge on the scene structure.

The next stages of this work will involve adapting the described materials for laser surgery applications. This will concern essentially the use of a high speed ( $\geq 200 \mathrm{fps}$ ) camera which will be connected to two image bundles through miniature GRIN lenses. It will be concern the study of the accuracy of the control law (using external sensor) and its stability (global proof of stability).

\section{REFERENCES}

[1] B. Babington, Ed., Report of the Huntarian Society. The London Medical Gazette, 1928.

[2] H. Eckel, S. Berendes, M. Damm, and J.P. Klusmann, "Suspension laryngoscopy for endotracheal stenting," Laryngoscope, vol. 113, pp. 11-15, 2003.

[3] A. Aloy and M. Grasl, "Endoscopy of larynx and trachea with rigid laryngo-tracheoscopes under superimposed high-frequency jet ventilation ", Endoscopy, 2013.

[4] F. Chaumette and S. Hutchinson, "Visual servo control, part 1: Basic approaches," IEEE Rob. and Autom. Mag., vol. 13, no. 1, pp. 82-90, 2006.

[5] Z. Li, W. Xie, and X. Tu, "Switching control of image based visual servoing with laser pointer in robotic assembly systems," in IEEE Int. C. on Syst., Man and Cyb., 2007, pp. 2383-2389.

[6] W. Xie, Z. Li, X. Tu, and C. Perron, "Switching control of image-based visual servoing with laser pointer in robotic manufacturing systems," IEEE T. on Ind. Elec., vol. 56, no. 2, pp. 520-529, 2009.

[7] P. Avanzini, E. Royer, B. Thuilot, and P. Martinet, "A global decentralized control strategy for urban vehicle platooning using monocular vision and a laser rangefinder," in Int. Conf. on Cont., Autom., Rob. and V., 2008, pp. 291-296.

[8] G. Karras, S. Loizou, and K. Kyriakopoulos, "A visual-servoing scheme for semi-autonomous operation of an underwater robotic vehicle using an imu and a laser vision system," in IEEE ICRA, 2010, pp. 5262-5267.

[9] M. Busack, G. Morel, and D. Bellot, "Breathing motion compensation for robot assisted laser osteotomy," in IEEE ICRA, 2010, pp. 45734578.

[10] A. Krupa, J. Gangloff, C. Doignon, M. F. de Mathelin, G. Morel, J. Leroy, L. Soler, and J. Marescaux, "Autonomous 3-d positioning of surgical instruments in robotized laparoscopic surgery using visual servoing," IEEE T. on Rob. and Autom., vol. 19, pp. 842-853, 2003.

[11] N. Andreff, S. Dembélé, B. Tamadazte, and Z. E Hussnain, "Epipolar geometry for vision-guided laser surgery," in Int. Conf. on Info. in Cont., Auto. and Rob., 2013, pp. 1-6.

[12] P. Rives, "Visual servoing based on epipolar geometry," in IEEE/RSJ IROS, vol. 1, 2000, pp. 602-607.

[13] N. Andreff, B. Espiau, and R. Horaud, "Visual servoing from lines," IJRR, vol. 21 (8), pp. 679-700, 2002.

[14] G. Mariottini, G. Oriolo, and D. Prattichizzo, "Image-based visual servoing for nonholonomic mobile robots using epipolar geometry," IEEE T. on Rob., vol. 23, no. 1, pp. 87-100, 2007.

[15] F. Alkhalil and C. Doignon, "Stereo visual servoing with decoupling control," in IEEE/RSJ IROS, 2012, pp. 1671-1676.

[16] E. Montijano, J. Thunberg, X. Hu, and C. Sagüès, "Epipolar visual servoing for multirobot distributed consensus," IEEE T. on Rob., vol. PP, no. 99, pp. 1-14, 2013.

[17] S. Lv, D. Zhang, and J. Gu, "Research of automatic needle locating based on stereo visual servoing," in 3rd Int. Conf. on Bio. Eng. and Info., vol. 4, 2010, pp. 1779-1783.

[18] B. Becker, R. MacLachlan, L. J. Lobes, and C. Riviere, "Semiautomated intraocular laser surgery using handheld instruments," Lasers in Surgery and Medicine, vol. 42, no. 3, pp. 264-273, 2010.

[19] R. Hartley and A. Zisserman, Multiple view geometry in computer vision, 2nd ed. Cambridge, United Kingdom: Cambridge University Press, 2006

[20] A. Shademan and M. Jagersand, "Three-view uncalibrated visual servoing," in IEEE/RSJ IROS, 2010, pp. 6234-6239.

[21] G. López Nicolás, J. Guerrero, and C. Sagüès, "Visual control through the trifocal tensor for nonholonomic robots," Rob. and Auton. Syst., vol. 58 , no. 2, pp. $216-226,2010$.

[22] G. Dagnino, L. S. Mattos, and D. G. Caldwell, "New software tools for enhanced precision in robot-assisted laser phonomicrosurgery," in 34th Annual Int. C. of the Eng. in Med. and Bio. Soc. (EMBC)., 2012.

[23] E. Marchand, F. Spindler, and F. Chaumette, "Visp for visual servoing: a generic software platform with a wide class of robot control skills," IEEE Rob. and Autom. Mag., vol. 12, no. 4, pp. 40-52, 2005. 